() И.Л. Плакса ${ }^{1,2}$, Н.Д. Мжаванадзе ${ }^{3}$, Р.Е. Калинин ${ }^{3}$, И.А. Сучков ${ }^{3}$, М.Ю. Бакунов ${ }^{4}$, В.Т. Кривихин ${ }^{5}$, С.А. Матвеев ${ }^{6}$, А.А. Исаев ${ }^{1}$, P.В. Деeв ${ }^{1,7}$

\author{
'Институт стволовых клеток человека, Москва \\ ${ }^{2}$ Московская городская онкологическая больница №62 Д3М, Москва \\ ${ }^{3}$ Рязанский государственный медицинский университет им. акад. И.П. Павлова, Рязань

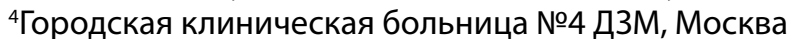 \\ ${ }^{5}$ Московский областной научно-исследовательский клинический институт им. М.Ф. Владимирского, Москва \\ ${ }^{6}$ Национальный медико-хирургический центр имени Н.И. Пирогова, Москва \\ ${ }^{7}$ Северо-Западный государственный медицинский университет им. И.И. Мечникова, Санкт-Петербург
}

ОБОСНОВАНИЕ. Синдром диабетической стопы (СДС) остается главной причиной нетравматической ампутации нижней конечности в мире. Даже при оказании исчерпывающей современной медицинской помощи в условиях специализированного центра у 10-15\% пациентов не удается добиться заживления язвенного дефекта вследствие выраженного ишемического компонента.

ЦЕЛЬ. Оценить безопасность и эффективность применения pI-VEGF165 у больных с СДС в составе комплексного лечения.

МЕТОДЫ. В пилотном исследовании приняли участие 35 пациентов с нейроишемической формой СДС, у которых отсутствовала техническая возможность выполнения реваскуляризирующей операции вследствие характера поражения дистального кровеносного русла, а проведение комплексной терапии не позволяло добиться заживления язвенного дефекта (международный идентификатор: NCT02538705). Продолжительность наблюдения составила 6 мес, с проведением плановых обследований через 1, 3 и 6 мес после введения первой дозы рI-VEGF165, в ходе которых выполнялись регистрация нежелательных явлений, оценка площади язвенного дефекта (первичный критерий эффективности), а также лодыжечно-плечевого индекса (ЛПИ), транскутанного напряжения кислорода (ТКНК) и выраженности нейропатического компонента (вторичные критерии эффективности).

РЕзУЛЬтАTЫ. Применение pl-VEGF165 в составе комплексного лечения позволило добиться заживления ран у 65,7\% больных с хроническими язвенными дефектами, сохранность целевой конечности составила $84 \%$. Проведение терапевтического ангиогенеза в составе комбинированной терапии обеспечило сокращение средней площади язвенных дефектов кожи с $3,6[1,0 ; 7,05] \mathrm{cm}^{2}$ до $0,0[0,0 ; 2,0] \mathrm{cm}^{2}(p=0,001)$, что сопровождалось увеличением показателя TКНК на 15\% - с 35,0 [29,5; 40,5] до 40,5 [36,0; 46,5] (p=0,005) и ЛПИ на 16\% - с 0,96 [0,82; 1,08] до 1,11 [0,85; 1,24] (p=0,062). Определялось снижение выраженности признаков диабетической нейропатии - показатели шкал НДС (нейропатический дисфункциональный счет) и НСС (нейропатический симптоматический счет) снизились соответственно с 6,5 $[5,75 ; 8,0]$ до $6,0[5,25 ; 7,0](p=0,004)$ и с $9,0[8,0 ; 13,5]$ до $8,0[7,0 ; 12,7](p=0,001)$. Осложнений, связанных с применением pl-VEGF165, зафиксировано не было.

ЗАКЛЮчЕНИЕ. Применение pl-VEGF165 в составе комплексного лечения пациентов с нейроишемической формой СДС безопасно и обеспечивает улучшение течения раневого процесса за счет улучшения перфузии тканей и коррекции нейропатического компонента.

КЛЮЧЕВЫЕ СЛОВА: синдром диабетической стопы; терапевтический ангиогенез; pl-VEGF165

\title{
PILOT STUDY OF THE SAFETY AND EFFICACY OF ANGIOGENIC THERAPY IN DIABETIC FOOT SYNDROME
}

(c) Igor L. Plaksa ${ }^{1,2}$, Nina D. Mzhavanadze”, Roman E. Kalinin ${ }^{3}$, Igor A. Suchkov³, Michail U. Bakunov ${ }^{4}$, Vyacheslav T. Krivichin ${ }^{5}$, Sergey A. Matveev ${ }^{6}$, Artur A. Isaev ${ }^{1}$, Roman V. Deev ${ }^{1,7}$

${ }^{1}$ Human Stem Cells Institute, Moscow, Russia

${ }^{2}$ Moscow City Oncology Hospital No 62, Moscow, Russia

${ }^{3}$ Ryazan State Medical University, Ryazan, Russia

${ }^{4}$ City Clinical Hospital No 4 of the Moscow Department of Health, Moscow, Russia

${ }^{5}$ M.F. Vladimirsky Moscow Regional Research and Clinical Institute, Moscow, Russia

${ }^{6}$ National Medical and Surgical Center named after N.I. Pirogov, Moscow, Russia

${ }^{7}$ North-Western State Medical University named after I.I. Mechnikov, Saint-Petersburg, Russia 
BACKGROUND: The syndrome of diabetic foot remains the main cause of non-traumatic amputation of the lower extremity in the world. Even with the provision of comprehensive medical care in the conditions of a specialized center, 10-15\% of patients do not succeed in healing the ulcerative defect due to the ischemic component.

AIMS: The objective of this study is evaluation of safety and efficacy of pl-VEGF165 transfer in patients with neuroischemic type of diabetic foot syndrome.

METHODS: The pilot study included 35 diabetic patients with neuroischemic foot ulcers (Wagner stage 1-2) who were not candidates for revascularization procedures (NCT02538705). The patients were closely monitored after repeated pl-VEGF165 intramuscular gene transfer $(2,4 \mathrm{mg})$ at 1,3 , and 6 months after treatment. The primary efficacy endpoint was the surface area of the ulcers (sq.cm), the secondary endpoints were transcutaneous oxygen tension (Tcp02), ankle-brachial index (ABI), neuropathy disability score (NDS), neuropathy symptoms score (NSS), and Michigan neuropathy screening instrument (MNSI). Adverse events were monitored throughout the study.

RESULTS: The use of pl-VEGF165 as part of complex treatment allowed to achieve wound healing in $65,7 \%$ of patients with chronic ulcerative defects, the safety of the target limb was $84 \%$. Carrying out therapeutic angiogenesis as a part of the combined therapy ensured a reduction in the average area of the resistant to treatment defects from 3.6 [1.0; 7.05$] \mathrm{cm}^{2}$ to $0.0[0.0 ; 2.0] \mathrm{cm}^{2}(p=0,001)$, which correlated with an increase in the TcPo2 index by $15 \%$ from 35 [29.5; 40.5] to $40.5[36.0 ; 46.5] \mathrm{mm} \mathrm{Hg}(p=p=0,005)$ and in the $A B I$ by $16 \%$ from $0.96[0.82 ; 1.08]$ to $1.11[0.85 ; 1.24](p=0,062)$. The decrease in the signs of diabetic neuropathy was determined - the scores of NSS scales and VAT decreased from 6,5 [5.75; 8.0) to $6.0[5.25 ; 7.0](p=0,004)$ and from $9.0[8.0 ; 13.5]$ to $8.0[7.0 ; 12.7](p=0,001)$, respectively. No adverse effects associated with the use of pl-VEGF165 were recorded.

CONCLUSIONS: Thus, preliminary results of the pilot study show that the use of pl-VEGF165 gene transfer in combination therapy allows for complete healing of neuroischemic diabetic foot ulcers in the majority of patients.

KEYWORDS: diabetic foot syndrome; therapeutic angiogenesis; pl-VEGF165

Синдром диабетической стопы (СДС) остается главной причиной нетравматической ампутации нижней конечности в мире [1]. Распространенность заболевания среди больных сахарным диабетом (СД) составляет от $2 \%$ до $10 \%$, частота нетравматических ампутаций 3,83 на 1 тыс. в год [2]. При этом средняя стоимость лечения пациента с нейроишемической формой СДС, по данным Эндокринологического научного центра (ЭНЦ), составляет 341,96 тыс. руб. [3]. Но даже при применении всех современных методов лечения в условиях специализированного центра у 10-15\% пациентов не удается добиться заживления язвенного дефекта вследствие ишемического компонента, нарушений репаративной регенерации и отсутствия возможности выполнения реваскуляризирующей операции, что значительно увеличивает риск развития инфекционных осложнений и ампутации. При этом арсенал терапевтических средств коррекции нарушения процесса репаративной регенерации тканей ограничен.

Одними из важных звеньев в патоморфогенезе структурных изменений тканей при различных формах диабетического поражения являются нарушение строения сосудистой стенки и, как следствие, выраженная депривация кровообращения в микроциркуляторном звене. Каскад последующих изменений, включая нейросенсорные и биомеханические, является в первую очередь следствием гипоксической гибели тканевых элементов. В этой связи одной из задач при реализации комплексного лечения таких пациентов является эффективное восстановление тканевой перфузии.

Клинические и морфологические исследования показали, что генная индукция ангиогенеза обеспечивает значимое улучшение функционального состояния боль- ных хронической ишемией нижних конечностей благодаря улучшению перфузии тканей за счет индукции ангиогенеза и возрастания количества сосудов микроциркуляторного русла $[4,5]$. В отдельных экспериментальных работах показан эффект фармакологической васкуляризации с использованием генноопосредованного терапевтического ангиогенеза при повреждении периферических нервов и диабетической нейропатии, что создает предпосылки для применения данного метода у больных с СДС с наличием как ишемического, так и нейропатического компонента [6]. Метаанализ клинических исследований различных генных конструкций с геном эндотелиального сосудистого фактора роста (Vascular Endothelial Growth Factor - VEGF) показал, что их применение не сопровождается увеличением частоты развития диабетической ретинопатии и ухудшения зрения [7].

В совокупности с данными по фармакокинетике это позволяет рассматривать препарат на основе плазмидного вектора как локальный индуктор ангиогенеза, что дает обоснование оценки эффективности применения генноопосредованной индукции ангиогенеза с целью улучшения репаративной регенерации тканей стопы при СДС.

\section{ЦЕЛЬ}

Выполнить предварительную оценку безопасности и эффективности геннотерапевтической конструкции, представляющей собой сверхскрученную форму плазмиды pl-VEGF165, кодирующей эндотелиальный сосудистый фактор роста (VEGF) в составе комплексного лечения пациентов с нейроишемической формой СДС в формате пилотного исследования. 


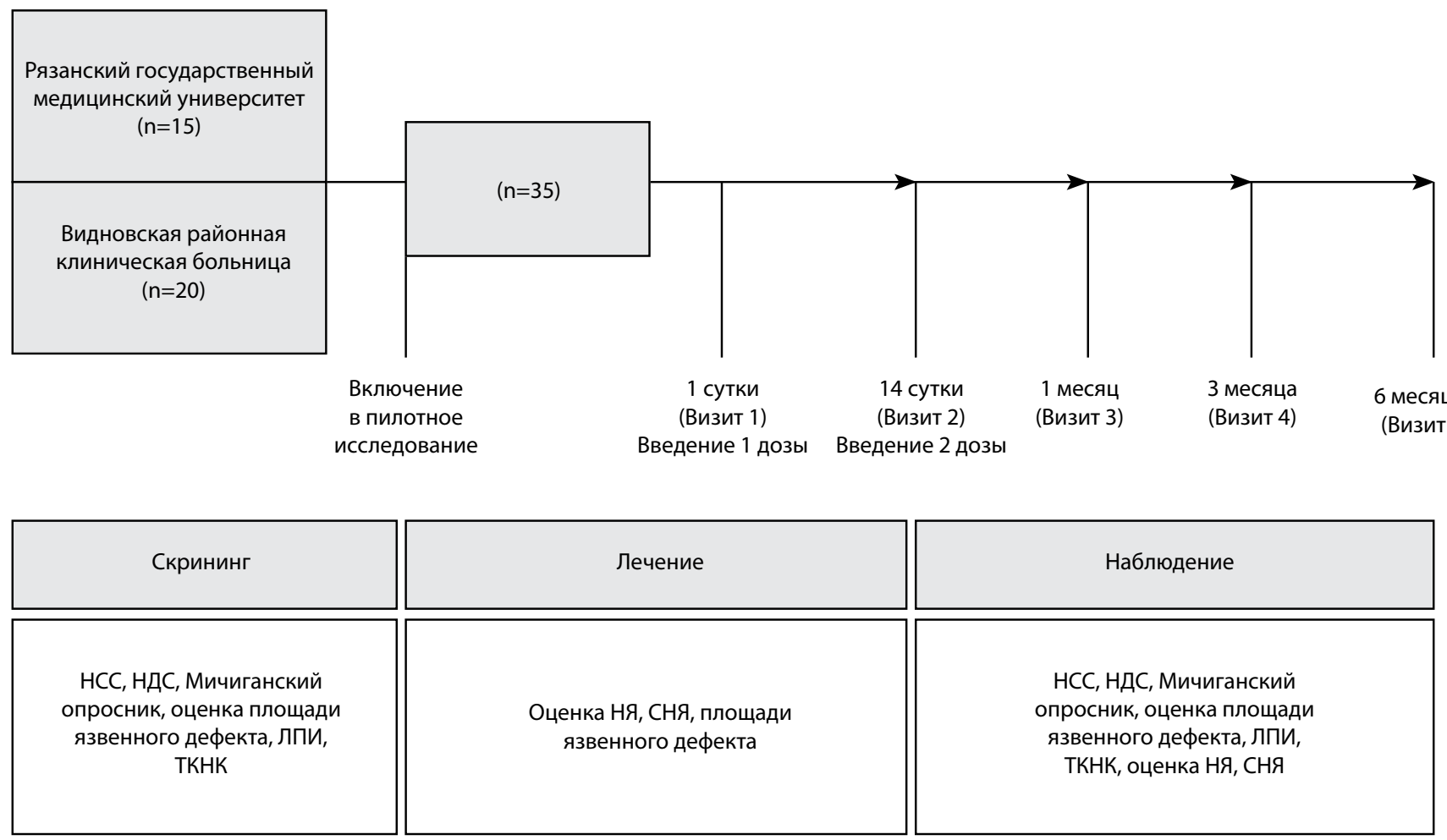

Рис. 1. Дизайн пилотного клинического исследования (схема): ЛПИ - лодыжечно-плечевой индекс; ТКНК - транскутанное напряжение кислорода; НДС - нейропатический дисфункциональный счет; НСС - нейропатический симптоматический счет; НЯ - нежелательные явления; СНЯ - серьезные нежелательные явления.

\section{МЕТОДЫ}

\section{Дизайн исследования}

Исследование было спланировано как интервенционное двухцентровое проспективное неконтролируемое неослепленное. Дизайн представлен на рис. 1.

\section{Критерии соответствия}

Пилотное клиническое исследование проводилось с участием больных с нейроишемической формой СДС $(n=35)$.

Критерии включения:

- возраст от 40 лет и старше;

- нейроишемическая форма СДС;

- длительно не заживающий язвенный дефект на фоне проводимой стандартной терапии (не менее 3 мес), ограниченный стопой, и отсутствие тенденции к заживлению;

- глубина язвенного дефекта 1-2 степени (по Вагнеру);

- отсутствие технической возможности выполнения реваскуляризирующей операции вследствие характера поражения дистального кровеносного русла или наличие реокклюзии на фоне выполненной не ранее чем за 6 мес до включения в исследование реваскуляризирующей операции;

- наличие гемодинамически значимых поражений магистральных артерий нижних конечностей по данным ангиографии и/или ультразвуковой допплерографии (УЗДГ);

- возможность осуществления ежедневного контроля гликемии с поддержкой уровня гликированного гемоглобина до 10\%;

- наличие письменного информированного согласия пациента.
Критерии невключения:

- нейропатическая форма СДС;

- выраженная деформация стопы;

- наличие гнойно-деструктивных поражений стопы (абсцесс, флегмона);

- недавняя (менее 3 мес) операция или эндоваскулярное вмешательство на артериях нижних конечностей или недавний (менее 3 мес) тромбоз глубоких вен нижних конечностей.

\section{Условия проведения}

Набор пациентов осуществлялся в Московской области на базе Государственного бюджетного учреждения здравоохранения Московской области «Видновская районная клиническая больница» и в Рязани на базе Рязанского медицинского университета. Набор пациентов в клиническое исследование происходил из числа больных, которые находились на стационарном лечении в условиях клиник этих учреждений.

\section{Продолжительность исследования}

Продолжительность исследования составила 6 мес с контрольными сроками обследования: при включении в исследование, 14, 30, 90 и 180 сут (см. рис. 1). В промежутках между визитами в исследовательский центр все пациенты находились на амбулаторном лечении у эндокринолога и хирурга по месту жительства; периодичность осмотров определялась индивидуально в зависимости от особенностей течения.

Описание медицинского вмешательства

Препарат сохранялся в виде лиофилизата, который разводился в 5 мл воды для инъекций непосредственно перед применением. Раствор вводился внутримышечно, 
в мышцы голени, двукратно, по 1,2 мг с интервалом 14 дней (суммарная доза 2,4 мг), в ходе 4-5 инъекций (согласно инструкции по применению). Пациенты получали стандартную комплексную терапию в соответствии с «Алгоритмами специализированной медицинской помощи больным сахарным диабетом» по ведению нейроишемической формы СДС, включавшую применение разгрузки конечности в виде разгрузочного полубашмака или индивидуальной разгрузочной повязки, местного лечения, контроля гликемии и т.д. [8]. Применение цилостазола и простагландинов было исключено не менее чем за 3 мес до включения в исследование.

\section{Основной исход исследования}

Выполнить предварительную оценку безопасности и эффективности pl-VEGF165 в составе комплексного лечения пациентов с нейроишемической формой СДС в формате пилотного исследования.

\section{Методы регистрации исходов}

В качестве первичного критерия эффективности была использована площадь язвенного дефекта, оцениваемая путем планиметрии (см²), вторичного - ТКНК, ЛПИ. Для оценки влияния ангиогенной терапии на выраженность диабетической нейропатии (ДН) в динамике были использованы стандартная шкала нейропатического симптоматического счета (НСC), нейропатический дисфункциональный счет (НДС), а также Мичиганский опросник для скрининга нейропатии. Стадия язвенного дефекта определялась по классификации Вагнера; согласно ей, при 1 стадии отмечается развитие поверхностной язвы (подкожные структуры не вовлечены); при 2 стадии - распространение язвы на глубоколежащие ткани без вовлечения костных структур. Выполнялась оценка количества летальных исходов и ампутаций за весь период наблюдения. Во время скрининга пациентам выполнялись ЭКГ, клинический и биохимический анализы крови, исследование свертывающей системы крови, анализ мочи с целью выявления сопутствующих и фоновых заболеваний.

\section{Этическая экспертиза}

Все пациенты перед включением в исследование были информированы о целях и задачах исследования, потенциальной пользе от участия и возможных рисках. Основанием для проведения пилотного клинического исследования явились разрешения локального этического комитета Рязанского медицинского государственного университета (№2 от 02.10.2014) и Видновской районной клинической больницы (№13 от 06.10.2014). Международный идентификатор на ClinicalGovTrial: NCT02538705.

\section{Статистический анализ}

Проведение статистического анализа было выполнено с использованием программы IBM SPSS Statistics 23.0.0.0. Для описания качественных данных рассчитывали абсолютные (n) и относительные значения (\%). Анализ соответствия вида распределения признака в каждой группе закону нормального распределения проводился с помощью критерия Шапиро-Уилка. Для выявления различий между измерениями показателей площади язвенного дефекта, ЛПИ, ТКНК, НСС, НДС, Мичиганского опросника нейропатии в разное время с учетом неболь- шой величины выборок применили критерий Уилкоксона, оценивающий медианную разность двух выборок, с уровнем значимости 0,05. Результаты были представлены с указанием медианы, первого и третьего квартилей, а также абсолютной величины достигнутого уровня значимости (р). Показатели возраста, продолжительности СД, существования язвенного дефекта и гликированного гемоглобина соответствовали нормальному распределению, их значения указаны в виде $\mathrm{M} \pm \mathrm{m}$.

\section{РЕЗУЛЬТАТЫ}

\section{Исходные характеристики пациентов пилотного} исследования

У большей части пациентов был длительный анамнез СД - в среднем 11,9 года (табл. 1). Продолжительность существования язвенного дефекта до включения в исследование составила 3,4 мес, что свидетельствует о хроническом течении раневого процесса и резистентности к проведению комплексного лечения. По глубине поражения преобладала 2 стадия по Вагнеру, которая определялась у $66 \%$ больных. При этом медиана площади раневого дефекта составила $3,6[1,0 ; 7,5]$ см² $^{2}$ рис. 2).

Таблица 1. Исходные показатели пациентов

\begin{tabular}{|c|c|}
\hline Показатель & Значение \\
\hline Число пациентов, n & 35 \\
\hline Средний возраст, год & $62,8 \pm 8,7$ \\
\hline Продолжительность СД, лет & $11,9 \pm 3,9$ \\
\hline Продолжительность существования & \\
\hline $\begin{array}{l}\text { язвенного дефекта до включения } \\
\text { в исследование, мес }\end{array}$ & $3,4 \pm 0,6$ \\
\hline Гликированный гемоглобин, \% & $8,6 \pm 1,2$ \\
\hline \multicolumn{2}{|c|}{ Распределение по полу, n (\%) } \\
\hline M & $47(67)$ \\
\hline ж & $23(32)$ \\
\hline \multicolumn{2}{|c|}{ Распределение по типу сахарного диабета, n (\%) } \\
\hline 1 тип & $2(6)$ \\
\hline 2 тип & $33(94)$ \\
\hline \multicolumn{2}{|c|}{ Локализация язвенного дефекта, n (\%) } \\
\hline Подошва & $14(40)$ \\
\hline Пальцы & $7(20)$ \\
\hline Тыл & $4(12)$ \\
\hline Пяточная область & $10(28)$ \\
\hline \multicolumn{2}{|c|}{ Стадия язвенного дефекта по Вагнеру, n (\%) } \\
\hline I & $12(34)$ \\
\hline II & $23(66)$ \\
\hline \multicolumn{2}{|c|}{$\begin{array}{c}\text { Предшествующее хирургическое (реваскуляризация) } \\
\text { лечение, n (\%) }\end{array}$} \\
\hline Да & $21(60)$ \\
\hline Нет & $14(40)$ \\
\hline \multicolumn{2}{|c|}{ Показатели критериев эффективности } \\
\hline Площадь язвенного дефекта, см² & $3,6[1,0 ; 7,05]$ \\
\hline ЛПИ, Ед & $0,96[0,82 ; 1,08]$ \\
\hline ТКНК, мм рт. ст. & $35[29,5 ; 40,5]$ \\
\hline НСС, баллы & $6,5[5,75 ; 8,0]$ \\
\hline НДС, баллы & $9,0[8,0 ; 13,5]$ \\
\hline Мичиганский опросник нейропатии & $7,67[6,2 ; 8,75]$ \\
\hline
\end{tabular}

Примечания: СД - сахарный диабет; ЛПИ - лодыжечно-плечевой индекс; ТКНК - транскутанное напряжение кислорода; НДС - нейропатический дисфункциональный счет; НСС - нейропатический симптоматический счет. 
Таблица 2. Распределение серьезных нежелательных явлений в клинической группе пилотного исследования.

\begin{tabular}{lc}
\hline \multicolumn{1}{c}{ Параметр } & Количество \\
\hline Большие ампутации & 3 \\
Малые ампутации & 2 \\
Онкологические заболевания & - \\
Сердечно-сосудистые осложнения & - \\
\hline
\end{tabular}

\section{Оценка безопасности}

Осложнений, связанных с применением pl-VEGF165, зарегистрировано не было. Развитие опухолей, нарушения зрения и иные патологические состояния, которые могли бы косвенно свидетельствовать об осложнениях ангиогенной терапии, не были выявлены на всех сроках обследования. Трем пациентам была выполнена ампутация нижней конечности на уровне бедра в связи с прогрессированием ишемии $(\mathrm{n}=2)$ и развитием анаэробной инфекции $(\mathrm{n}=1)$; двум - в пределах стопы. К концу наблюдения у 4 из 5 пациентов раны зажили первичным натяжением, у одного пациента определялось сохранение раневого дефекта. Таким образом, сохранность целевой конечности к концу 6 мес наблюдения составила 84\% (табл. 2).

\section{Оценка эффективности}

Применение pl-VEGF165 в составе комбинированного лечения позволило добиться полного закрытия ран у 66\% пациентов $(n=23)$, несмотря на длительное предшествующее существование язвенного дефекта на фоне проведения стандартного комплексного лечения. При этом заживление большей части из них $(\mathrm{n}=15)$ наблюдалось на сроке 3 мес. Напротив, отсутствие положительного эффекта к этому времени являлось неблагоприятным прогностическим фактором - как правило, у этой категории больных открытая рана сохранялась и к концу исследования или была выполнена ампутация (табл. 3).

К концу 1-го месяца после введения первой дозы pl-VEGF165 у 68\% пациентов ( $n=24)$ отмечалось уменьшение площади менее чем на 30\%, при этом среднее значение снизилось с 3,6 [1,0; 7,05] $\mathrm{cm}^{2}$ до $3,35[1,0 ; 6,1] \mathrm{cm}^{2}$ $(p=0,006)$. В течение следующих 2 мес у большей части больных отмечалась положительная динамика репа- ративной регенерации тканей в области язвы, что привело к полному заживлению ран у 42\% больных $(n=15)$. Медиана площади язвенного дефекта к концу 3 мес сократилась с $3,6[1,0 ; 7,05]$ см $^{2}$ до $1,05[0,0 ; 4,1](p=0,001)$, к концу исследования - до 0,0 [0,0; 2,0] см². Средняя скорость заживления на протяжении всего исследования составила 2,1 мм²/сут. При этом скорость заживления в течение первых 30 сут была 0,9 мм²/сут, с последующим увеличением до 3,4 мм²/сут с 30 до 90 сут. Начиная с $90-x$ суток было отмечено снижение скорости заживления до 1,7 мм²/сут. Учитывая продолжительное время существования раны до включения в исследование, такая тенденция сокращения площади и эпителизации свидетельствует об изменении условий репаративной регенерации тканей (см. рис. 2).

ЛПИ к концу исследования возрос на 19\%, с $0,96[0,82 ; 1,08]$ до $1,16[0,85 ; 1,24](p=0,062)$, что, по-видимому, объясняется изменением периферического сосудистого сопротивления на фоне проводимого лечения. Несмотря на наличие небольшой положительной динамики показателя на фоне лечения, различия значений не достигли статистической достоверности. У 3 пациентов, несмотря на наличие ишемического компонента вследствие поражения дистального кровеносного русла,

$\mathrm{CM}^{2}$

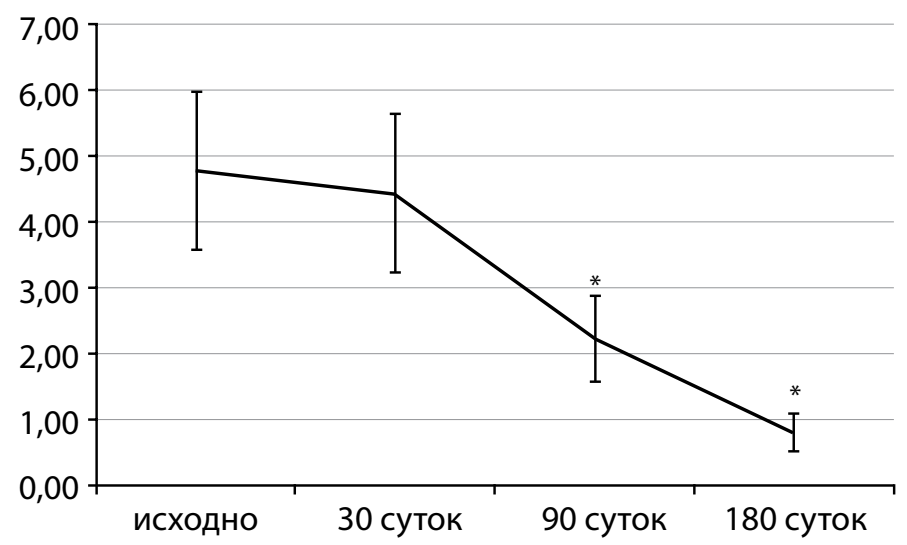

Рис. 2. Динамика площади язвенного дефекта $\left(\mathrm{cm}^{2}\right)$ : * различие между показателем и предыдущим значением статистически значимо $(\mathrm{p}<0,01)$.

Таблица 3. Динамика заживления язвенного дефекта, n (\%)

\begin{tabular}{lccc}
\hline \multicolumn{1}{c}{ Исход лечения } & Через 1 мес & Через 3 мес & Через 6 мес \\
\hline Полное заживление & $1(2,8)$ & $15(42)$ & $23(65,7)$ \\
Уменьшение площади язвенного дефекта на 70\% & $4(11,4)$ & $4(11,4)$ & $3(8,5)$ \\
Уменьшение площади язвенного дефекта на 50\% & $2(5,8)$ & $5(14,2)$ & $3(0)$ \\
Уменьшение площади язвенного дефекта менее 50\%, но более 30\% & $2(5,7)$ & $24(68,5)$ & $6(17)$ \\
Уменьшение площади язвенного дефекта менее 30\% & $2(5,8)$ & $0(0)$ & $3(8,5)$ \\
Увеличение площади язвенного дефекта & $0(0)$ & $1(2,8)$ & $2(0)$ \\
Ампутация выше колена & $0(0)$ & $1(2,8)$ & $3(5,8)$ \\
Малая ампутация в пределах стопы & &
\end{tabular}


данный показатель изначально был выше 1,0, что может быть связано с выраженным кальцинозом сосудистой стенки. На фоне применения терапевтического ангиогенеза определялось улучшение перфузии тканей стопы, что выражалось в возрастании напряжения кислорода с 35,0 [29,5; 40,5] до 40,5 [36,0; 46,5] мм рт.ст. (p=0,005), при этом наибольший прирост наблюдался в течение первых 3 мес.

На фоне применения ангиогенной терапии через 6 мес отмечалось умеренное, но значимое снижение выраженности клинических признаков ДН - показатели НСС и НДС снизились соответственно с 6,5 [5,75; 8,0] до $6,0[5,25 ; 7,0](p=0,001)$ и с $9,0[8,0 ; 13,5]$ до $8,0[7,0 ; 12,7]$ $(p=0,004)$. Объективно пациенты отмечали снижение выраженности судорог и чувства онемения. В отдельных случаях было отмечено появление температурной чувствительности через неделю после второй инъекции plVEGF165 и частичное восстановление болевой чувствительности (табл. 4).

\section{ОБСУЖДЕНИЕ}

Мировой опыт применения ангиогенных препаратов у больных СДС насчитывает лишь несколько исследований, поэтому основной задачей настоящей работы была оценка безопасности генноопосредованной индукции ангиогенеза у пациентов с СД. Впервые этот подход был реализован в ходе 1-2 фазы клинических исследований Ad-5PDGF-B (GAM501) у 15 пациентов с хроническими язвами при нейропатической форме СДС, которым геннотерапевтическая конструкция на основе вируса наносилась на язвенный дефект в составе коллагенового геля. Это не сопровождалось развитием нежелательных реакций, но при этом полного закрытия на фоне комбинированного лечения удалось добиться у 12 из 15 пациентов $[9,10]$. В другом исследовании применение плазмиды с геном HGF (VM202) у пациентов с критической ишемией нижних конечностей обеспечило достоверное увеличение частоты заживления дефектов кожных по- кровов стопы и не сопровождалось развитием осложнений. При этом исследование показало, что применение индукции ангиогенеза было наиболее эффективно у пациентов с СД, которые составили 60\% участников [11]. Отечественные исследования с участием больных хронической ишемией нижних конечностей показали, что у пациентов с фоновым СД локальная индукция ангиогенеза в нижних конечностях не сопровождается развитием нежелательных явлений [12]. Таким образом, результаты настоящего пилотного исследования подтверждают ранее накопленные доказательства безопасности ангиогенной терапии у пациентов с СД.

Площадь язвенного дефекта была выбрана как основной критерий эффективности потому, что в обычной клинической практике уменьшение размера язвы и/или ее полное закрытие часто определяет, достигнут ли успех лечения пациента с СДС или нет [13]. Однако использование этого показателя ассоциировано с применением жестких критериев включения, определяющих, пациенты с какими именно язвенными дефектами могут быть включены в исследование, так как скорость и исход репаративной регенерации зависят от большого количества факторов. Например, результаты исследования с участием 261 больного СДС показал, что среднее время заживления язвенного дефекта составляет 49 дней, однако наличие раневой инфекции или остеомиелита сопровождается увеличением сроков заживления в среднем до 83 и 115 дней. В то же время у пациентов с преобладанием ишемического компонента среднее время заживления составило 58 дней, а при его отсутствии - 46 [14]. Ввиду этого включение в настоящее пилотное исследование пациентов с неглубокими язвенными дефектами 1-2 стадии по Вагнеру было обусловлено стремлением нивелировать многочисленные факторы, которые влияют на процесс репаративной регенерации, и достичь, таким образом, максимальной объективности при оценке влияния генноопосредованной индукции ангиогенеза на сроки заживления язвенного дефекта.

Таблица 4. Результаты оценки вторичных критериев эффективности в пилотном исследовании

\begin{tabular}{|c|c|c|c|}
\hline Показатель & Исходно & 3 мес & $6 \mathrm{mec}$ \\
\hline лпи & $\begin{array}{c}0,96[0,82 ; 1,08] \\
(n=35)\end{array}$ & $\begin{array}{c}1,04[0,79 ; 1,1] \\
p=0,121 \\
(n=31)\end{array}$ & $\begin{array}{c}1,11[0,85 ; 1,24] \\
p=0,062 \\
(n=29)\end{array}$ \\
\hline TKHK & $\begin{array}{c}35,0[29,5 ; 40,5] \\
(n=29)\end{array}$ & $\begin{array}{c}40,0[34,5 ; 46,0] \\
p<0,001 \\
(n=17)\end{array}$ & $\begin{array}{c}40,5[36,0 ; 46,5] \\
p=0,005 \\
(n=20)\end{array}$ \\
\hline НДС & $\begin{array}{c}9,0[8,0 ; 13,5] \\
\quad(n=30)\end{array}$ & - & $\begin{array}{c}8,0[7,0 ; 12,7] \\
p=0,001 \\
(n=20)\end{array}$ \\
\hline $\mathrm{HCC}$ & $\begin{array}{c}6,5[5,75 ; 8,0] \\
\quad(n=30)\end{array}$ & - & $\begin{array}{c}6,0[5,25 ; 7,0] \\
p=0,004 \\
(n=20)\end{array}$ \\
\hline Мичиганский опросник & $\begin{array}{c}7,8[6,2 ; 8,7] \\
(n=16)\end{array}$ & - & $\begin{array}{c}7,0[5,75 ; 8,0] \\
p=0,285 \\
(n=8)\end{array}$ \\
\hline
\end{tabular}

Примечания: ЛПИ - лодыжечно-плечевой индекс; ТКНК - транскутанное напряжение кислорода; НДС - нейропатический дисфункциональный счет; НСС - нейропатический симптоматический счет. 
Результаты настоящего исследования показали, что наибольшая скорость сокращения язвенного дефекта наблюдалась в интервале между 30 и 90 сут - то есть через месяц после введения pl-VEGFA, что может быть обусловлено отсроченным действием генной индукции ангиогенеза. Напротив, снижение средней скорости заживления в интервале времени между 90 и 180 сут, а также отсутствие заживления к концу 3-го месяца лечения может являться проявлением ограниченного срока действия препарата вследствие разрушения плазмидной конструкции и, как следствие, ограниченного периода экспрессии целевого белка - VEGF. В то же время отдельные исследования выделяют прогностические факторы заживления язвенного дефекта, которые могут быть ассоциированы с исходными условиями репаративной регенерации. Например, уменьшение площади язвенного дефекта на 50\% к концу 4-й недели является прогностическим критерием заживления раны через 12 нед. Напротив, отсутствие заживления к этому сроку сопровождается достоверным увеличением частоты ампутации [15]. Таким образом, уже к концу 3-го месяца после введения pl-VEGFA можно выделить категорию пациентов, у которых применение ангиогенной индукции может оказаться неэффективным ввиду не только ограниченного периода экспрессии целевого белка, но и исходных изменений сосудистой стенки, нарушений репаративной регенерации, которые настолько выражены, что не могут быть скорректированы при помощи генной индукции ангиогенеза. Однако эта гипотеза, безусловно, требует подтверждения на более крупных группах пациентов.

Показатель ЛПИ широко применяется в повседневной практике как экспресс-метод диагностики заболеваний периферических артерий у пациентов с облитерирующим атеросклерозом. В отдельных работах показано, что этот показатель может быть также использован как прогностический фактор заживления язвенного дефекта у больных СДС. Так, метаанализ 11 клинических исследований показал, что ЛПИ менее 0,5 достоверно чаще ассоциирован с вероятностью резистентного течения раневого процесса и ампутации нижней конечности [16]. Несмотря на небольшое увеличение показателя на фоне генноопосредованной индукции ангиогенеза, различия не достигли статистической достоверности, что может быть обусловлено малым объемом выборки в данном исследовании. По-видимому, в рамках данной работы применение лПИ как критерия эффективности оказалось малоинформативным ввиду исходно высокого значения показателя ЛПИ из-за выраженного кальциноза артерий. Вследствие этого интерпретация увеличения среднего показателя ЛПИ затруднена - ответ на вопрос, является ли это следствием применения ангиогенной терапии или нет, требует проведения более крупных исследований.

В отдельных работах было показано, что применение генной терапии способно обеспечить снижение выраженности проявлений ДН. В первом плацебо-контролируемом исследовании с участием больных СД было показано, что введение плазмиды с геном VEGF в проекции седалищного, малоберцового и большеберцового нервов обеспечивает значимое улучшение показателя шкалы SS и не приводит к развитию нежелательных явлений [17]. Активным компонентом препарата VM202 является плазмида с генами двух изоформ HGF. B ходе 1-й фазы клинического исследования с участием 12 пациентов с болевой формой ДН была доказана безопасность и хорошая переносимость ангиогенной терапии с использованием дозировок 4 мг, 8 мг и 16 мг [18]. Во 2-й фазе двойного слепого плацебо-контролируемого клинического исследования с участием 100 пациентов с болевой формой ДН использовались дозировки 16 мг и 32 мг. Препарат вводился двукратно с интервалом 2 нед в процессе 16 и 32 инъекций соответственно. В качестве первичного критерия эффективности использовался среднесуточный показатель шкалы боли относительно базального уровня, вторичных - показатель визуальной аналоговой шкалы, качества жизни, а также интраэпидермальная плотность нервных волокон по данным щипковой биопсии на уровне бедра и голени с интервалом в 6 мес. Применение генного препарата не сопровождалось увеличением частоты развития нежелательных явлений, при этом по первичному критерию было достигнуто статистически значимое различие [19]. Результаты настоящего исследования показали, что на фоне применения генноопосредованной индукции ангиогенеза наблюдалось снижение выраженности проявлений ДН, что выражалось в улучшении показателей шкал НСС и НДС. Как известно, субъективные части шкал могут улучшаться вследствие купирования воспаления и уменьшения отека тканей.

В совокупности полученные данные свидетельствуют о том, что генная индукция ангиогенеза pl-VEGF165 может быть использована в составе комплексного лечения пациентов с СДС, в том числе при невозможности выполнения реваскуляризации и нарушении репаративной регенерации язвенного дефекта - введение препарата было безопасно и не сопровождалось развитием нежелательных явлений. Результаты показали, что pl-VEGFA воздействует как на ишемический, так и нейропатический компонент заболевания. Это позволяет рассматривать его как средство патогенетической терапии, которое обладает многокомпонентным эффектом в отношении данной нозологии. Однако для трансляции полученных данных на общую популяцию пациентов с СДС необходимо проведение крупных рандомизированных исследований.

\section{Ограничения исследования}

Ограничением данного исследования является открытый дизайн, что увеличивает вероятность появления систематической ошибки в интерпретации симптомов и признаков в ведении пациентов, возникающей, когда лечение пациента заранее известно. Выбор подобного дизайна обусловлен тем, что генноиндуцированный ангиогенез ранее никогда не применялся у данной категории пациентов, ввиду чего основной целью работы была предварительная оценка безопасности и эффективности на небольшом количестве пациентов. Также ограничением исследования является то, что запланированные методы инструментальной оценки некоторым пациентам применялись не на всех сроках обследования, что было обусловлено техническими сложностями (отсутствие комплектующих, специалистов и т.д.). 


\section{ЗАКЛЮЧЕНИЕ}

Результаты показали, что применение генноопосредованной индукции ангиогенеза pl-VEGF165 у пациентов с СДС не сопровождалось развитием осложнений, связанных с индукцией ангиогенеза. На фоне применения pl-VEGF165 у большей части пациентов удалось достичь заживления язвенных дефектов, несмотря на отсутствие технической возможности выполнения реваскуляризирующей операции вследствие характера поражения дистального кровеносного русла. Это сопровождалось улучшением перфузии мягких тканей стопы по данным ТКНК и снижением выраженности нейропатического компонента. Таким образом, генноопосредованная индукция ангиогенеза у пациентов с СДС может являться одним из компонентов комплексного лечения этой категории пациентов. Дальнейшее подтверждение эффективности этого подхода требует проведения рандомизированных сравнительных исследований на больших группах пациентов.

\section{ДОПОЛНИТЕЛЬНАЯ ИНФОРМАЦИЯ}

Источник финансирования. Исследование выполнено при финансовой поддержке компании-разработчика ПАО «Институт стволовых клеток человека».

Конфликт интересов. А.А. Исаев - акционер компании-разработчика ПАО «Институт стволовых клеток человека»; Р.В. Деев и И.Л. Плакса - сотрудники ПАО «Институт стволовых клеток человека».

Участие авторов. Плакса И.Л. - проведение мониторинга клинического исследования; Мжаванадзе Н.Д. - набор пациентов и сбор данных; Сучков И.А. - разработка дизайна исследования; Калинин Р.Е. - анализ данных, полученных в ходе исследования; Бакунов М.Ю. - набор пациентов в клиническое исследования и анализ данных; Кривихин В.Т. - разработка дизайна исследования; Матвеев С.А. - консультирование на этапе разработки дизайна протокола клинического исследования; Исаев А.А. - координация проекта; Деев Р.В. - координация проекта. Все авторы внесли существенный вклад в проведение исследования и подготовку статьи, прочли и одобрили финальную версию до публикации.

\section{СПИСОК ЛИТЕРАТУРЫ | REFERENCES}

1. Frykberg RG, Zgonis T, Armstrong DG, et al. Diabetic foot disorders. A clinical practice guideline (2006 revision). J Foot Ankle Surg. 2006;45(5 Suppl):S1-66. doi: https://doi.org/10.1016/S1067-2516(07)60001-5

2. Green MF, Aliabadi Z, Green BT. Diabetic foot: evaluation and management. South Med J. 2002;95(1):95-101. doi: https://doi.org/10.1097/00007611-200201000-00019

3. Галстян Г.Р., Сергеева С.В., Игнатьева В.И., и др. Клиникоэкономическое обоснование стоимости квоты на лечение пациентов с синдромом диабетической стопы // Сахарный диабет. — 2013. — №3. - C. 71-83. [Galstyan GR, Sergeeva SV, Ignat'eva VI, et al. Clinical and economical grounds of budgetary quotation for patients with diabetic foot syndrome. Diabetes Mellitus. 2013;(3):71-83. (In Russ.)] doi: https://doi.org/10.14341/2072-0351-820

4. Deev R, Bozo I, Mzhavanadze N, et al. pCMV-vegf165 intramuscular gene transfer is an effective method of treatment for patients with chronic lower limb ischemia. J Cardiovasc Pharmacol Ther. 2015;20(5):473-482. doi: https://doi.org/10.1177/1074248415574336

5. Деев Р.В., Калинин Р.Е., Червяков Ю.В., и др. Отдаленные результаты применения pl-VEGF165 при хронической ишемии нижних конечностей вследствие облитерирующего атеросклероза // Кардиология и сердечно-сосудистая хирургия. 2015. - T. 8. — №4. - C. 43-49. [Deev RV, Kalinin RE, Chervyakov $\mathrm{YV}$, et al. Long-term results of pl-VEGF165 intramuscular gene transfer in patients with atherosclerotic chronic lower limb ischemia. Cardiology \& cardiovascular surgery. 2015;8(4):43-49. (In Russ.)]

6. Saaristo A, Tammela T, Farkkila A, et al. Vascular endothelial growth factor-C accelerates diabetic wound healing. Am J Pathol. 2006;169(3):1080-1087. doi: https://doi.org/10.2353/ajpath.2006.051251

7. Gupta R, Tongers J, Losordo DW. Human studies of angiogenic gene therapy. Circ Res. 2009;105(8):724-736. doi: https://doi.org/10.1161/CIRCRESAHA.109.200386.

8. Галстян Г.Р., Токмакова А.Ю., Егорова Д.Н., и др. Клинические рекомендации по диагностике и лечению синдрома диабетической стопы // Раны и раневые инфекции. - 2015. T. 2. - №3. - C. 63-83. [Galstyan GR, Tokmakova AY, Egorova DN, et al. Clinical guidelines for diagnosis and treatment of diabetic foot syndrome. Wounds and wound infections. 2015;2(3):63-83. (In Russ.)]

9. Mulder G, Tallis AJ, Marshall VT, et al. Treatment of nonhealing diabetic foot ulcers with a platelet-derived growth factor gene-activated matrix (GAM501): results of a phase 1/2 trial. Wound Repair Regen. 2009;17(6):772-779. doi: https://doi.org/10.1111/j.1524-475X.2009.00541.x

10. Phase $2 \mathrm{~b}$ study of GAM501 in the treatment of diabetic ulcers of the lower extremities (MATRIX) [Internet]. ClinicalTrials.gov; 2020 [cited 2007 June 27]. Available from: https://clinicaltrials.gov/ct2/show/NCT00493051

11. Safety and efficacy study of VM202 in the treatment of chronic non-healing foot ulcers [Internet]. ClinicalTrials.gov; 2020 [cited 2015 September 30]. Available from: https://clinicaltrials.gov/ct2/show/ NCT02563522?term=VM202\&rank=4

12. Deev R, Plaksa I, Bozo I, Isaev A. Results of an international postmarketing surveillance study of pl-VEGF165 safety and sufficacy in 210 satients with peripheral arterial disease. Am J Cardiovasc Drugs. 2017:17(3):235-242. doi: https://doi.org/10.1007/s40256-016-0210-3

13. Bolton LL. Quality randomized clinical trials of topical diabetic foot ulcer healing agents. Adv Wound Care (New Rochelle). 2016;5(3):137-147. doi: https://doi.org/10.1089/wound.2014.0571

14. Milne T, Schoen D, Bower $V$, et al. Times of diabetic foot ulcers: investigating the influence of infection and peripheral arterial disease. J Diabet Foot Compl. 2013;5(2):29-38.

15. Sheehan $P$, Jones $P$, Caselli $A$, et al. Percent change in wound area of diabetic foot ulcers over a 4-week period is a robust predictor of complete healing in a 12-week prospective trial. Diabetes Care. 2003;26(6):1879-1882. doi: https://doi.org/10.2337/diacare.26.6.1879

16. Brownrigg JR, Hinchliffe RJ, Apelqvist J, et al. Performance of prognostic markers in the prediction of wound healing or amputation among patients with foot ulcers in diabetes: a systematic review. Diabetes Metab Res Rev. 2016;32 Suppl 1:128-135. doi: https://doi.org/10.1002/dmrr.2704

17. Ropper AH, Gorson KC, Gooch CL, et al. Vascular endothelial growth factor gene transfer for diabetic polyneuropathy: A randomized, double-blinded trial. Ann Neurol. 2009;65(4):386-393. doi: https://doi.org/10.1002/ana.21675

18. Ajroud-Driss S, Christiansen M, Allen JA, Kessler JA. Phase 1/2 open-label dose-escalation study of plasmid DNA expressing two isoforms of hepatocyte growth factor in patients with painful diabetic peripheral neuropathy. Mol Ther. 2013;21(6):1279-1286. doi: https://doi.org/10.1038/mt.2013.69

19. Kessler JA, Smith AG, Cha BS, et al. Double-blind, placebo-controlled study of HGF gene therapy in diabetic neuropathy. Ann Clin Transl Neurol. 2015;2(5):465-478. doi: https://doi.org/10.1002/acn3.186 


\section{ИНФОРМАЦИЯ ОБ АВТОРАХ [AUTHORS INFO]}

*Плакса Игорь Леонидович [Igor L. Plaksa]; адрес: Россия, 117312, Москва, ул. Губкина, д. 3 [address: 3 Gubkina street, Moscow, 117312 Russian Federation]; ORCID: https://orcid.org/0000-0001-6600-0933; e-mail: i.plaksa2014@yandex.ru

Мжаванадзе Нина Джансуговна, К.M.H. [Nina D. Mzhavanadze, MD, PhD];

ORCID: https://orcid.org/0000-0001-5437-1112; eLibrary SPIN: 7757-8854; e-mail: nina_mzhavanadze@mail.ru

Сучков Игорь Александрович, д.м.н., професcop [lgor A. Suchkov, MD, PhD, Professor];

ORCID: https://orcid.org/0000-0002-1292-5452; eLibrary SPIN: 6473-8662; e-mail: suchkov_med@mail.ru

Калинин Роман Евгеньевич, д.м.н., професcop [Roman E. Kalinin, MD, PhD, Professor];

ORCID: https://orcid.org/0000-0002-7894-3055; eLibrary SPIN: 5009-2318; e-mail: kalinin-re@yandex.ru

Бакунов Михаил Юрьевич [Michail U. Bakunov];

ORCID: https://orcid.org/0000-0002-5511-0149; eLibrary SPIN: 9213-6072; e-mail: mbakunov@bk.ru

Кривихин Вячеслав Тимофеевич, к.м.Н., професcop [Vyacheslav T. Krivichin, MD, PhD, Professor];

ORCID: https://orcid.org/0000-0001-8408-1984; eLibrary SPIN: 2196-2279; e-mail: krivihin.slava@yandex.ru

Матвеев Сергей Анатольевич, д.м.н., професcop [Sergey A. Matveev, MD, PhD, Professor];

ORCID: https://orcid.org/0000-0003-0237-608X; eLibrary SPIN: 6228-0722; e-mail: glebcenter@mail.ru

Исаев Артур Александрович [Artur A. Isaev]; eLibrary SPIN: 2623-0638; e-mail: art.isaev@gmail.com

Деев Роман Вадимович, к.м.н., доцент [Roman V. Deev, MD, PhD, associate professor];

ORCID: https://orcid.org/0000-0001-8389-3841; eLibrary SPIN: 2957-1687; e-mail: romdey@gmail.com

\section{ЦИТИРОВАТЬ:}

Плакса И.Л., Мжаванадзе Н.Д., Калинин Р.Е., Сучков И.А., Бакунов М.Ю., Кривихин В.Т., Матвеев С.А., Исаев А.А., Деев Р.В. Пилотное исследование безопасности и эффективности ангиогенной терапии при синдроме диабетической стопы // Сахарный диабет. - 2019. — Т. 22. — №6. — C. 559-567. doi: https://doi.org/10.14341/DM9644

\section{TO CITE THIS ARTICLE:}

Plaksa IL, Mzhavanadze ND, Kalinin RE, Suchkov IA, Bakunov MY, Krivichin VT, Matveev SA, Isaev AA, Deev RV. Pilot study of the safety and efficacy of angiogenic therapy in diabetic foot syndrome. Diabetes Mellitus. 2019;22(6):559-567. doi: https://doi.org/10.14341/DM9644 